SCIENCE \& TECHNOLOGY
Journal homepage: http://www.pertanika.upm.edu.my/

\title{
A Combined Analytical Method for Intelligent Control of Friction Damped Structures
}

\author{
Kamyar Gharra, Karen Khanlari* and Jafar Asgari Marnani \\ Department of Civil Engineering, Faculty of Technology and Engineering, Central Tehran Branch, \\ Islamic Azad University, Tehran, Iran
}

\begin{abstract}
Controlling structures and increasing the prognosis of their behaviour before natural disasters are the most critical issues in structural engineering. To that end, predicting the destructive effects of earthquakes on both acceleration and displacement of structures would be beneficial. This paper suggests an intelligent control system that realises simultaneous control of acceleration and displacement parameters. There are two modules in the system. First, the preserving module aims to estimate the crisis thresholds of acceleration and displacement based on the historical seismic data of each area. Second, the processing module finds the optimum value of the slip load of the friction damper so that both acceleration and displacement are controlled. We introduce an analytical method based on a matrix analysis approach and heuristic algorithm (MAHA) as a core of the processing module. MAHA would analyse the structure response, and the friction damper would determine the optimum slip load. The numerical and software simulation results for various one-bay and two-bay steel structures show that the proposed intelligent control system applies to multiple frictions damped structures under different earthquake records. In addition, a control level of $80 \%$ in acceleration and displacement of structures is achieved compared to an uncontrolled state. Moreover, the mentioned system enables the engineers to find appropriate friction dampers during the design of structures.
\end{abstract}

ARTICLE INFO

Article history:

Received: 22 December 2020

Accepted: 19 April 2021

Published: 22 September 2021

DOI: https://doi.org/10.47836/pjst.29.4.05

E-mail addresses:

kamyar.gharra75@gmail.com (Kamyar Gharra)

karen.khanlari@gmail.com (Karen Khanlari)

j_asgari@iauctb.ac.ir (Jafar Asgari Marnani)

* Corresponding author
Keywords: Analytical modelling, control system, damped friction structure, multi-degree of freedom

\section{INTRODUCTION}

There has been a difference in views among scientists in the earthquake and structural engineering (Domenico et al., 2020; Rashidi et al., 2020). Structural scientists focused 
on strengthening structures, as well as the optimal design of buildings under earthquakes loads. The design of new buildings, including seismic restraint systems, is one of the new structural topics in recent decades (Nizic \& Mestrovic, 2011; Majd et al., 2019; Sanghai $\&$ Pawade, 2020). These systems only act against various seismic vibrations without withstanding any static loads. In seismic restraint systems, dampers help to increase the loss of seismic energy entering the building. As a result, a desirable and durable structure can be achieved. The structures behave safely against all kinds of dynamic earthquake loads (Shaw, 1986; Pall, 1996; Fateh et al., 2016).

Friction dampers are classified as passive control systems and effectively control vibrations and reduce seismic responses. One of the most critical parameters in designing friction-damped structures is slip load. It has a significant impact on seismic and the level of non-elastic behaviour of the structure (Pasquin, 2004; Bhaskararao \& Jangid, 2006).

In this regard, various researches have been done in recent years, including "The Seismic Reinforcement of Steel Frame Buildings using A Friction Damper by Lee S. et al. (2008). Scientists in earthquakes assess the response of acceleration and displacement between the floors of each structure. It was shown that the dissipated energy is effectively lost for a 10-story building with the proper distribution of dampers, and the structural behaviour is linear. In another study, Lee H. et al. (2008) investigated a base shear on designing a frictional damper system in a structure with elastic behaviour. They showed that the sliding load of the damper was determined with the shear of the floors in the initial bending frame. Also, it was shown that the distribution of slip load in proportion to the shear of the floor was effective. Hence, it could reduce the relative displacement of the floors by comparing the slip load's uniform distribution.

Some investigations were conducted on optimising the performance of friction dampers within the structures. The use of heuristic algorithms in this domain emerged. Apostolakis and Dargush (2010) studied the optimal seismic design of steel bending frames with metal dampers or additional friction with a restraint system. They used a genetic algorithm to find the slip load of the structure. In order to evaluate the response of the optimised structure, they used the sum of the squares of the relative displacement or the acceleration of the floors. They compared three examples of bending frame models in pre and post improvement mode with the addition of dampers. The results showed that after the optimal design, the above response parameters were reduced. Also, the distribution of floor acceleration and relative displacement of floors was more uniform. In 2013 and 2015, two types of research were conducted by Fallah and Honarparst (2013) and Feliciano (2015). The purpose of these studies was to investigate the optimal slip load in multi-story buildings. They distributed the slip load in a structure using two modes; uniform and non-uniform. In both modes, the sum of slip loads in a structure was considered the same. Then, the optimal slip load was obtained in each case using a multivariate optimisation method. 
In order to optimally design the location of friction dampers, several studies were conducted. For example, Lopez and Miguel (2015) found a method using the FireFly algorithm and the finite difference method on a ten-story building. Therefore, it was possible to obtain a suitable location for the damper to minimise the maximum displacement within the structure.

Kim and An (2016) conducted a study on nonlinear static and dynamic analyses of friction damped structures. They found the optimum position of friction dampers by genetic algorithm. They concluded that maximum roof displacement and the inter-story drift ratio were reduced by $30 \%$ and $40 \%$, respectively. Perez et al. (2017a \& 2017b) also worked on an optimisation method to control the failure probability of the friction-damped structure. The failure probability of the structure was defined by the criterion of increasing the amount of displacement between floors from $1 \%$ of the height of the first floor. The results for a three-story structure showed that optimising the damper location and frictional force could reduce the risk of failure by up to $80 \%$.

Miguel and Lopez (2018) presented an approach to simultaneously optimise the frequency domain's frictional location and friction damper parameters. One of the advantages of Miguel's study was a significant reduction in computational time. The design was based on a six-story building. Variables such as force and displacement of the friction damper have been investigated. However, the results showed that the average displacement decreased by approximately $82 \%$. Palacios et al. (2020) worked on protecting multiple adjacent buildings by using distributed damping systems. They reported about $50 \%$ in the overall inter story-drift of $40 \%$ in the overall acceleration peak-value.

As mentioned in the literature overview, they used the approximated model to identify the behaviour of friction damped structures. Moreover, they are limited to a specific region and individual structure parameters. This paper intends to advance previous research and propose an intelligent control system equipped with a combined analysis method. The novelty of the proposed control system is that it is designed parametric and more accurate. That means by varying the structures and earthquakes in each area, and we can put the relevant parameters. The system inputs include the number of stories, earthquake specifications, number of friction dampers, number of degrees of freedoms, allowable levels of acceleration, and displacements on that area. The outputs are optimum slip load, controlled acceleration, and displacement responses. The main objectives of the proposed idea are as follows:

- Implementing the intelligent control system that is knowledge-based, flexible, and in any geographical area. The system is composed of preserving and processing modules. The acceleration and displacement thresholds are analysed based on the historical seismic earthquake records of that region. Then, the processing module finds the optimum value of slip load so that acceleration and displacement are controlled jointly. 
- Using an optimisation method in the core of the intelligent control system. It can work adaptively based on the variation of structural parameters and earthquake records.

- Combining matrix analysis of the reduced-order equation of motion, exact modelling of each friction joint, as well as a heuristic algorithm. This analytical approach gives the optimum value of slip load.

- Estimating the controlled response of structures during the structure design. It is based on the outputs of the intelligent control system and the appropriate friction damper in each structure.

The discussion of this paper will start by addressing the problem formulation. Second, the conceptual diagram of the intelligent control system and systematic parameters are described. Third, introducing the proposed MAHA as an effective solution for finding the optimum slip load of the friction damper. Fourth, the discussion will be based on the numerical results and simulation scenarios before being concluded.

\section{METHODS}

This study is conducted to realise an intelligent control system that applies to various structures in each area. First, the proposed system's substantial parameters, such as acceleration and displacement of structures, would be kept at an acceptable level. Then, the idea is proposed and formulated before introducing the combined analytical method to find the response of various structures.

\section{Problem Formulation}

Figure 1 depicts the conceptual diagram of the proposed intelligent control system. The system consists of two modules. First, the preserving module provides the set of processes to store, index, and access information. The information includes the data of seismic stations that measure the earthquake records. The data mining step can be started by creating a historical records of earthquakes in a specific area. In this step, the maximum acceleration and displacement levels on an area are detected.

Second, to process the acceleration and displacement levels on which the structures experienced minimum damage. Then, threshold levels are calculated and put into the processing module. In this module slip load of each story is defined as a decision variable. The primary role of this module is to find the optimum slip load of friction damper for designing an appropriate friction damper for that structure. To this end, first, the structure is analysed by the proposed matrix analysis method. Then acceleration and displacement responses are extracted. Finally, these responses are used as inputs of the optimisation method. 


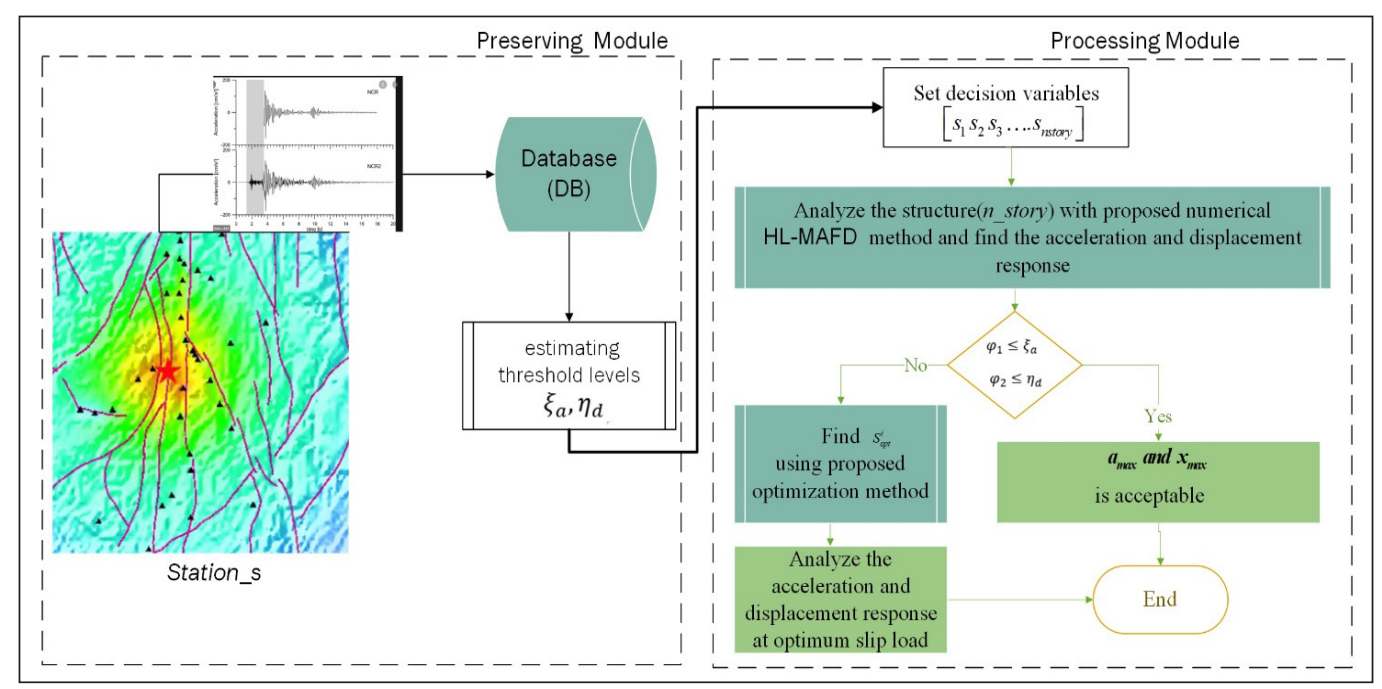

Figure 1. Conceptual diagram of the proposed intelligent control system

The output of the proposed intelligent control system is a controlled acceleration and displacement and an optimum value of slip load. This system can be set up in any area and can apply to various structures and earthquake records. To form a database, we have used the Seismic station data of Iran (For example, Manjil and Tabas) and El-Centro records.

The systematic parameters of this investigation are:

- Seismic station of Tabas and Manjil, (Station_s)

- SQL Database and Mathcad as a software medium, (DB)

- Server Core i7-8700 CPU at 3.20 GHz, 16 GB RAM, and a 480GB SSD hard drive, (processing module)

- Medium- size structures, $\left(n_{\text {story }}\right)$

The optimisation method raised to find the optimal slip load is defined in Equation 1a. This definition includes design variables, objective function, and constraints. Minimising the joint acceleration and displacement is considered an objective function. It is due to the behaviour of structures in the natural environment. The optimisation methods are designed in such a way to support various behaviour of structures under different earthquakes. Structures with higher natural frequency and short natural periods suffer higher acceleration. On the contrary, structures with lower natural frequency and long natural periods suffer higher displacement. So, in the proposed intelligent control system the objective function is designed based on the above considerations. Design variables include the force of friction dampers in different floors, threshold level of acceleration and displacement, relations $(i)$ and (ii), as well as the minimum and maximum slip loads; constraint (iii).

Find $\vec{S}, \vec{S}=\left[S_{1} S_{2} S_{3} \ldots . S_{\text {nstory }}\right]$ 
Minimize $U(S)=\left[\boldsymbol{a}_{\max } \times \boldsymbol{x}_{\max }\right]$

\section{Subject to}

(i) $\varphi_{1} \leq \xi_{a}$

(ii) $\varphi_{2} \leq \eta_{d}$

(iii) $S^{\text {Min }} \leq S_{\text {opt }}^{i} \leq S^{\text {Max }}$

$S_{1} S_{2} S_{3} \ldots S_{n s t o r y}$ : decision variables that show slip load of each story;

$\boldsymbol{a}_{\max }:$ maximum acceleration of structure;

$\boldsymbol{x}_{\text {max }}$ : maximum displacement of structure;

$S^{\text {Max }}$ : maximum value of slip load;

$S_{\text {opt }}^{i}$ : minimum value of slip load;

$S_{\text {opt }}^{i}$ : optimum value of slip load;

$\varphi_{1}=\frac{\left|a_{\max _{-} d}\right|}{a_{\max }}:$ ratio of the maximum acceleration with damper to without damper;

$\varphi_{2}=\frac{\left|x_{\max _{\_} d}\right|}{x_{\max }}$ : ratio of the maximum displacement with damper to without damper;

$\eta_{d}:$ threshold level of displacement;

$\xi_{a}:$ threshold level of acceleration.

$U(S)$ is the objective function that consists of displacement and acceleration, which is actually a non-linear function. The functions of $x_{\max }$ and $a_{\max }$ do not closed-form formulation (Equation 1b). In fact, displacement and acceleration response are derived from the general equation of motion in Equation 2 in the following sections.

$$
U(S)=x_{\max }(s) \cdot a_{\max }(s)=x_{\max }(s) \cdot \frac{d}{d t^{2}} x_{\max }(s)
$$

Solving this equation is very complicated for MDOF (Multi Degree of Freedom) structures, especially the high-rise. For this purpose, the combined MAHA (Matrix Analysis and Heuristic Algorithm) is introduced based on the proposed HL-MAFD (Hysteresis Less-Matrix Analysis of Friction Damped structure) and a heuristic algorithm. The whole structure is first analysed by the proposed HL-MAFD algorithm, which is based on the basic principles of matrix analysis. In this step, the acceleration and displacement response will be obtained. Then, the optimum slip load in which both acceleration and displacement are controlled is obtained by a heuristic method. Figure 1 illustrates the conceptual flowchart of the proposed MAHA that performs based on the proposed HL-MAFD and SGA (search group algorithm). 


\section{Solving the Optimisation method}

MAHA will find the appropriate slip load to meet the joint controlled response of acceleration and displacement of the structure. It is a combination of HL-MAFD, numerical matrix analysis, and a heuristic SGA. First, the initial response of acceleration and displacement of the structure is obtained from the HL-MAFD. In this method, an accurate system stiffness matrix is achieved to form the general equation of motion. Generally, when analysing friction braces in standard software, the shape of hysteresis curve is selected according to the manufacturer's specifications. Then the whole structure analysis is done according to the link of the structure (Bhaskararao \& Jangid, 2006).

The innovation of the proposed approach compared to the previous ones is that the frictional element is first analysed numerically. The analysis is based on the basic principles of structural matrix analysis, structural dynamics, and relationships governing the general equation of motion at each time. In general, this approach is provided with a model for analysing frictional damper behaviour without the need for mechanical hysteresis curves. First, the motion general equation of the structure is formed. Then, the response of acceleration and displacement of the structure would be extracted. This response was obtained from solving the optimised formulation. The proposed method is a combination of the SGA (search group algorithm) and matrix analysis approach.

\section{Numerical HL-MAFD}

Figure 2 shows a structure equipped with a friction damper with $N$ degrees of freedom (NDOF). Therefore, it is necessary to define additional freedom to perform dynamic analysis for friction joints in braces. By defining the degrees of freedom in the axial direction in Figure 2, the relative velocity of the two ends of the connection can be determined. Then, the frictional force can be determined with the help of the relative velocity of the two ends. After determining the frictional force, the force relations feedback is examined and applied as an external force to the system. Finally, the structure is analysed.

Regarding the methods used in SAP (Structural Analysis Program) or ETABS (Extended Three-Dimensional Analysis

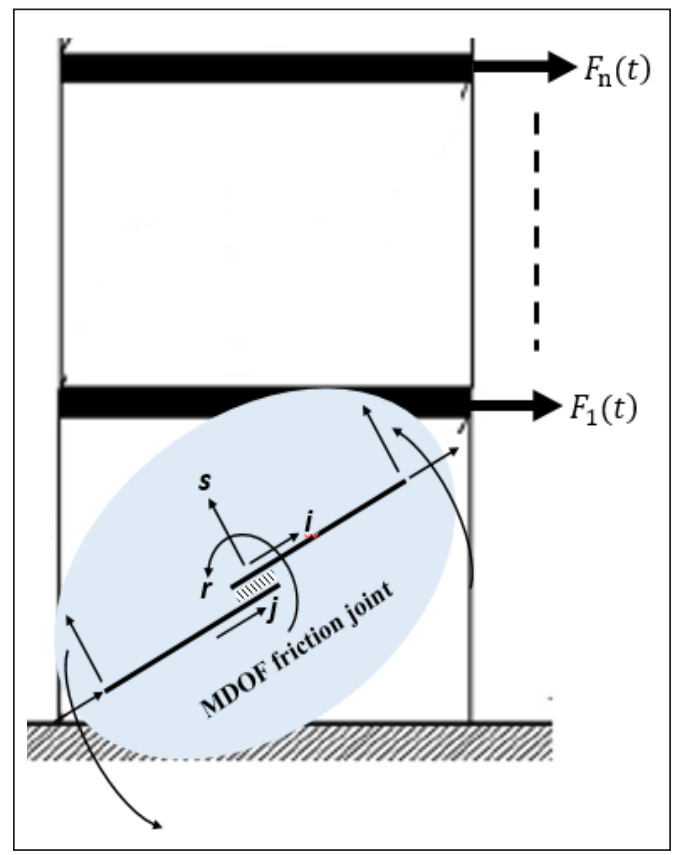

Figure 2. Structural model of a multi-degree of freedom with friction damper 
of Building System) software, the proposed numerical analysis approach for structures with frictional elements do not require the use of an ideal hysteresis curve provided by the manufacturer. Therefore, in the proposed method, the behaviour of the damper is not predetermined according to its specifications. Instead, the system stiffness matrix is accurately obtained by considering the stiffness matrix of system components in proportion to master and salve freedom degrees.

The degrees of freedom considered in frictional elements include two degrees of axial freedom in the direction of frictional and one degree of vertical freedom. Also, one degree of rotational freedom is considered to prevent the friction link from breaking. The general motion equation is shown in Equation 2.

$$
M \overrightarrow{x(t)}+K \overrightarrow{x(t)}+f(t)=F(t)
$$

Where $f(t)=-\mu S$.sign $(x)$ in which $f(t)$ is Friction force; $S$ is slip load, and $\mu$ is a friction coefficient. $M$ : mass matrix with the size of NDOF $\times$ NDOF; $K$ : stiffness matrix with the size of NDOF $\times$ NDOF; NDOF $=m+s$; total Number of DOFs; $m$ : number of master DOFs; $s$ : number of slave DOFs; $x(t)$ : acceleration response vector; $\dot{x}(t)$ : velocity response vector; $\dot{x}(t)$ : displacement response vector; $F(t)$ : external force vector.

The degrees of freedom are divided into master and slave to perform static compression. It should be noted that dynamic loading is not performed on the slave degrees of freedom. In this case, the general motion equation can be partitioned into master and slave to form Equation 3.

$$
\left[\begin{array}{cc}
M_{m m} & O_{s m} \\
O_{s m} & O_{s s}
\end{array}\right]\left[\begin{array}{l}
x_{m} \\
\cdot \\
x_{s}
\end{array}\right]+\left[\begin{array}{cc}
K_{m m} & K_{m s} \\
K_{s m} & K_{s s}
\end{array}\right]\left[\begin{array}{l}
x_{m} \\
x_{s}
\end{array}\right]+\left[\begin{array}{l}
O_{m} \\
f_{s}
\end{array}\right]=\left[\begin{array}{c}
F_{m} \\
O_{s}
\end{array}\right]
$$

$x_{m}$ : Displacement vector of master DOFs

$x_{s}$ : Displacement vector of slave DOFs

$K_{m m} \cdot K_{m s} \cdot K_{s m}$ and $K_{s s}$ : Partitions of the Stiffness matrix

$M_{m m}$ : Main partition of the Mass matrix

$O_{m s} \cdot O_{s m}$ and $O_{s s}:$ Null partitions of the Mass matrix

$F_{m}$ : External forces vector on master DOFs

$f_{s}$ : Friction forces vector on slave DOFs

$O_{s}:$ Null partition of Friction forces vector

$O_{s}$ : Null partition of External forces vector

By expansion of Equation 3 we will have Equation 4a and 4b:

$$
M_{m m} x_{m}+K_{m m} x_{m}+K_{m s} x_{s}=F_{m}
$$




$$
K_{s m} x_{m}+K_{s s} x_{s}=f_{s}
$$

Arranging Equation $4 \mathrm{~b}$, the displacement vector of slave DOFs can be represented in terms of stiffness, displacement vector of master DOFs, and friction forces vector on slave DOFs as Equation 4c.

$$
x_{s}=K_{s s}^{-1}\left(f_{s}-K_{s m} x_{m}\right)
$$

By applying the result of Equation 4c into Equation 4a, we obtain Equation 5.

$$
M_{m m} \ddot{x}_{m}+\left(K_{m m}-K_{m s} K_{s s}^{-1} K_{s m}\right) x_{m}=F_{m}-K_{m s} K_{s s}^{-1} f_{s}
$$

So, comparing Equation 5 with traditional general motion equation, we can extract the condensed or reduced mass and stiffness matrices as Equation 6a and $6 \mathrm{~b}$.

$$
\begin{aligned}
& \boldsymbol{M}^{*}=M_{m m} \\
& K^{*}=K_{m m}-K_{m s} K_{s s}^{-1} K_{s m}
\end{aligned}
$$

Where $K_{m m}, K_{m s}, K_{s s}, K_{s m}, K_{s s}$ are submatrices of $K_{s y s}$ as shown in Equation (17). The reduced external force vector is defined as Equation 7.

$$
\boldsymbol{F}^{*}=F_{m}-K_{m s} K_{s s}^{-1} f_{s}
$$

So, general motion equation of a system can be redefined based on master DOFs as Equation 8.

$$
\boldsymbol{M}^{*} x_{m}+\boldsymbol{K}^{*} x_{m}=F^{*}
$$

In the condensed general motion equation, the external force vector of master DOFs, $F_{m}$, is the seismic forces (i.e., earthquake). In the same way, the vector of forces related to slave DOFs, $F_{s}$, is the vector in which frictional forces are placed. To achieve the velocity of slave DOFs, $\dot{x}_{s}$, considering Equation $4 \mathrm{~b}$, we can obtain by differentiation of this vector as Equation 9.

$$
\begin{aligned}
& x_{s}=K_{s s}{ }^{-1} f_{s}-K_{s s}{ }^{-1} K_{s m} x_{m} \\
& \frac{d}{d t} x_{s}=K_{s s}{ }^{-1} \frac{d}{d t} f_{s}-K_{s s}{ }^{-1} K_{s m} \frac{d}{d t} x_{m}
\end{aligned}
$$


Hence, we can consider that the vector of the frictional forces of slave DOFs is equal to zero $\left(\frac{d}{d t} f_{s} \cong 0\right)$. So, we can approximately redefine the velocity vector of slave DOFs as Equation 10.

$$
\dot{x}_{s} \cong-K_{s s}{ }^{-1} K_{s m} \dot{x}_{m}
$$

Considering Equation 10, we can define the velocity of slave DOFs in terms of the master DOFs. In fact, by this important relationship we can solve the condensed general motion equation in a lower degree.

\section{Proposed MAHA}

HL-MAFD numerical analysis approach was described in the previous section. So, we can find the answer to the acceleration and displacement of any structure based on the reduced general motion equation with the exact stiffness matrix. As mentioned in the conceptual diagram of Figure 1, the goal of the optimisation method is to find the value of acceleration and displacement of the structure in the optimum slip load. Figure 3 addresses

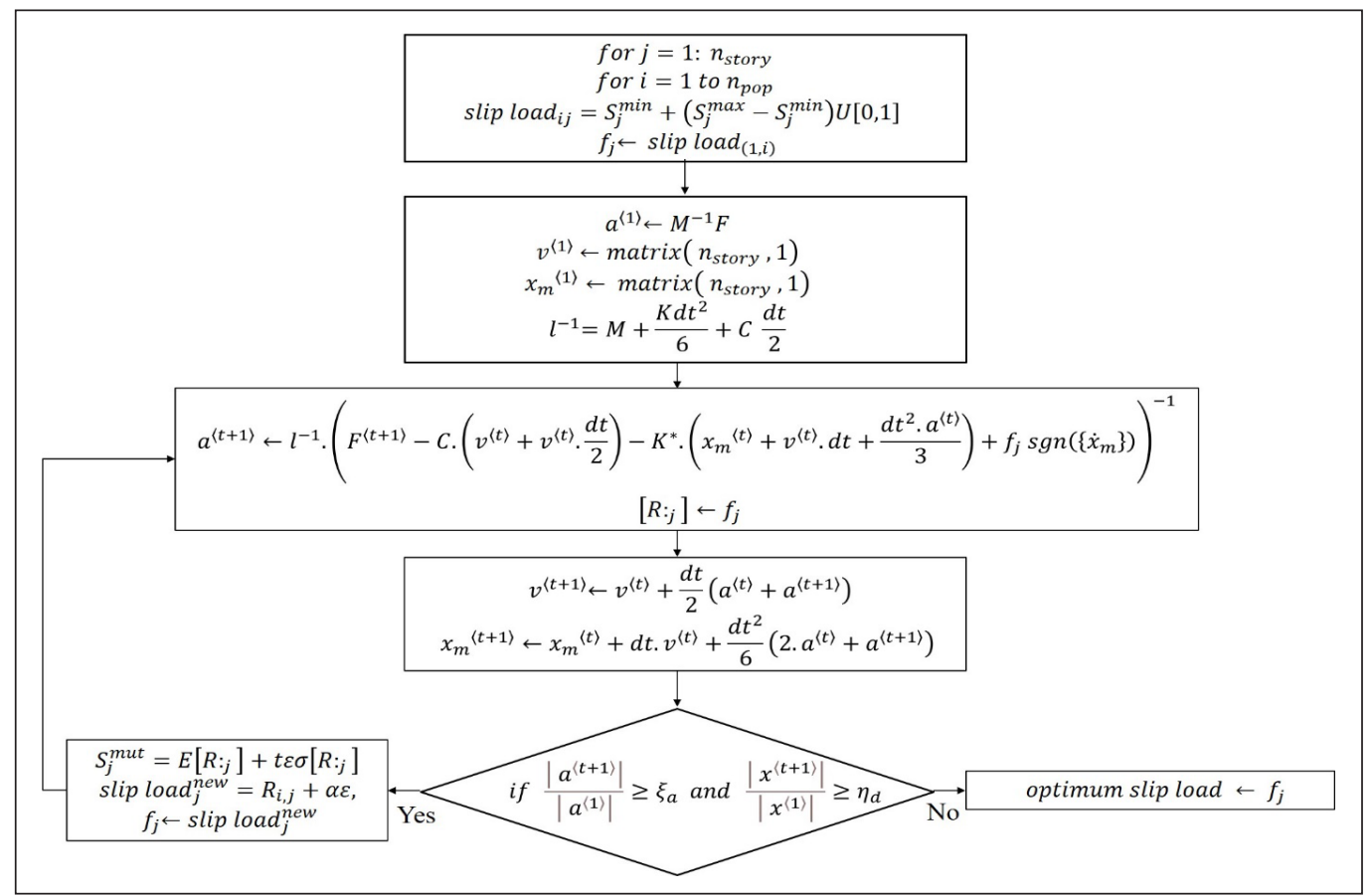

Figure 3. Flowchart of pseudo code of the MAHA

Note. $n_{\text {story }}$ : number of stories; $n_{\text {pop }}$ : number of initial populations of slip load; $U[1,0]$ : uniform variable; $E$ and $\sigma$ : mean and variance; slip load ${ }_{i j}$ :generated population; $R:_{j}$ j'th column in search matrix; $\varepsilon$ : random variable; $t$ control coefficient of new variable; $\boldsymbol{C}$ : damping matirx $C \propto$ eigenvalue $\left(\boldsymbol{M}^{-1} \boldsymbol{K}\right) ; . \gamma, \beta$ : New marks coefficient 
the functionality of the MAHA that is a combination of the proposed HL-MAFD and the heuristic SGA algorithm.

As can be seen in Figure 3, optimisation variables are the slip load of the structure. First, for each structure floor, an initial population is created for the slip load $\left(n_{p o p}\right)$. Then, acceleration and displacement values of the structure are obtained using the general motion equation obtained by the HL-MAFD at each time step. Also, the calculation of a sample stiffness matrix, $\boldsymbol{K}$, is given in Equation 16. Then, the acceleration and displacement output matrices are evaluated with the conditions mentioned in the optimisation method, defined as the minimum acceleration and displacement. If the condition does not satisfy, the amount of mutation will be determined for the slip load. Then, a new family will be formed for it. After that, the acceleration and displacement values will be calculated for the new values of slip load. This step repeats so that the condition is realised and the optimal slip load is obtained. Any variable that removes the objective function from the target range is more likely to be removed and replaced by a new variable. The values that are close to the optimal value would be stored in the search set $\xi_{a}$, and the slip load that meets the $\xi_{a}, \eta_{d}$ would be the final one.

\section{RESULTS AND DISCUSSION}

Several scenarios have been studied and measured to verify the proposed analytical optimisation method. As mentioned in this paper, the proposed analytical algorithm HL-MAFD is used to obtain the structural response to extract the structural stiffness matrix. Then, the general equation of motion with reduced orders is solved at the optimum value of slip load. A 4-story structure with a friction damper is considered to depict the performance of a proposed approach. Two cases are analysed: an optimised slip load and a non-optimised. We show that the acceleration and displacement responses of the structure with optimised slip load can be controlled considerably comparing the non-optimised.

Figure 4 shows the desired structure, degrees of freedom, and nodes based on the proposed algorithm. A load of $30 \mathrm{kN}$

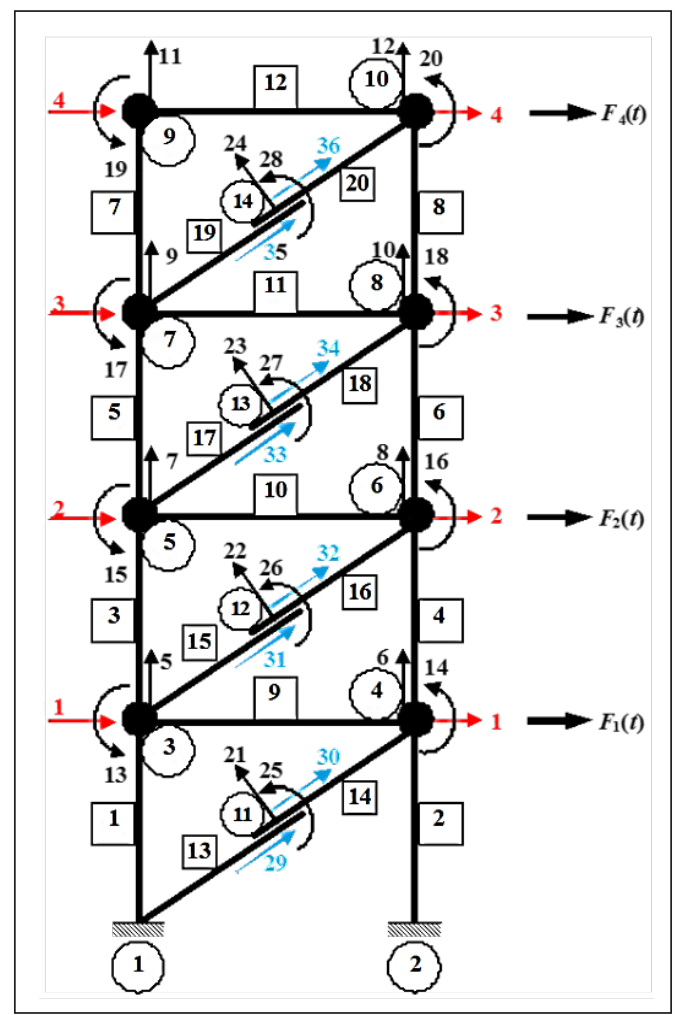

Figure 4. 4-story structure analysis under proposed method 
is applied to each node. Also, the seismic hazard zonation factor is considered $0.2 \mathrm{~g}$. The cross-sections of the structure are designed based on the static distribution of force.

Simulations are done by Mathcad mathematical software. Various earthquake records with specific PGA are used as an external force. PGA (Peak Ground Acceleration) means maximum ground acceleration that occurred during earthquake at a location. PGA is equal

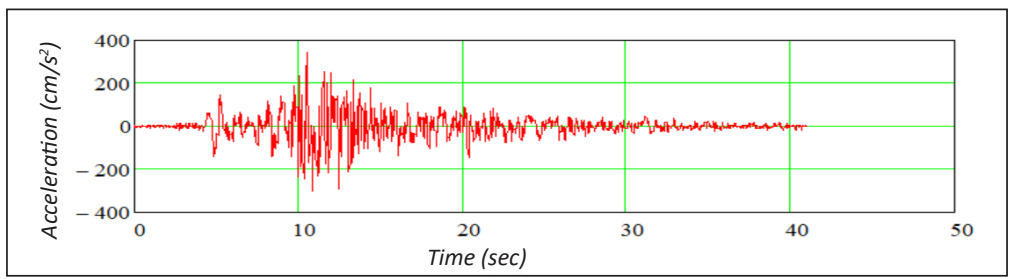

(a)

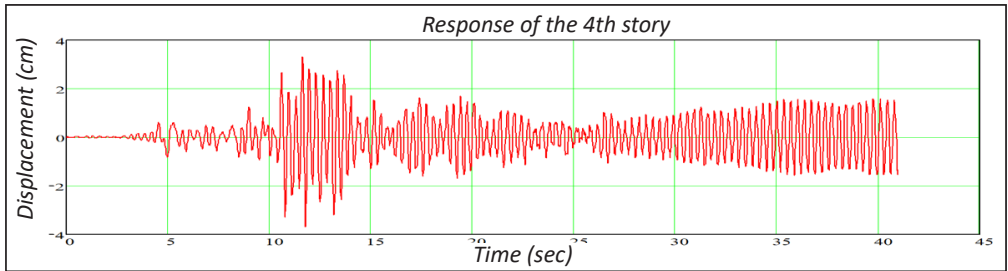

(b)

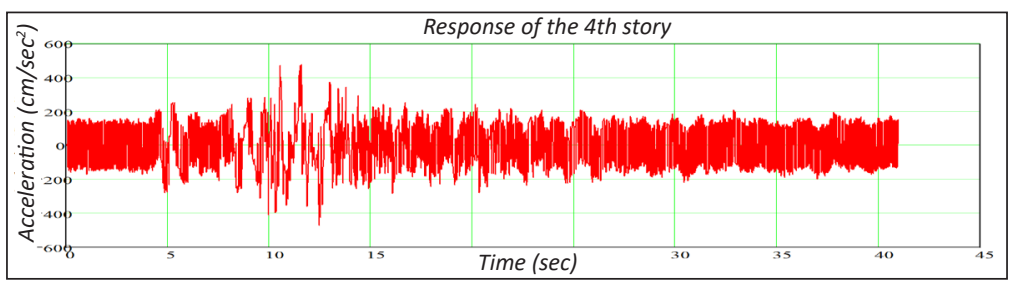

(c)

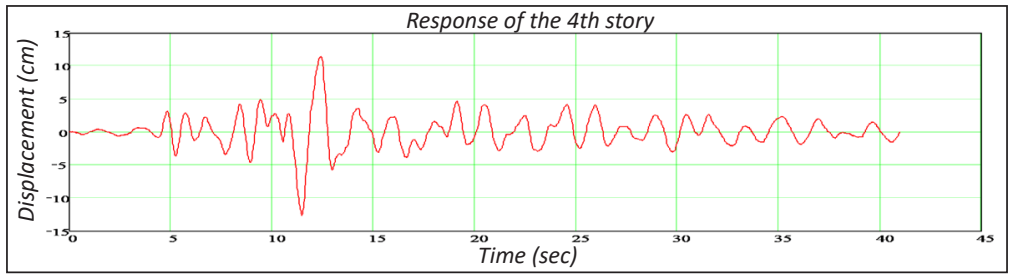

(d)

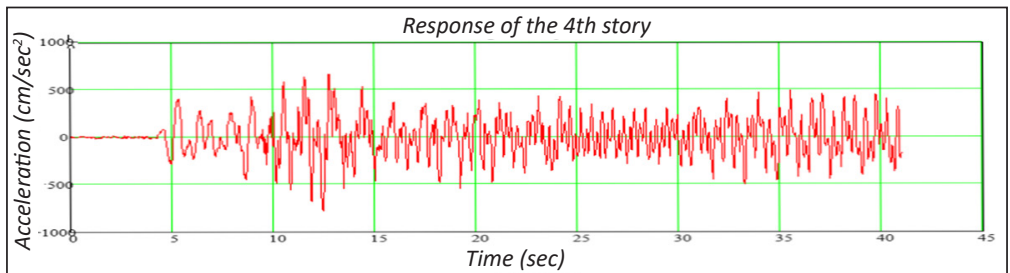

(e)

Figure 5. Measuring results of a four-story structure: (a) Tabas records; (b) Displacement with optimal slip load; (c) Acceleration with optimal slip load; (d) Displacement with non-optimised slip load; (e) Acceleration with non-optimised slip load 
to the amplitude of the largest absolute acceleration recorded on an accelerogram during earthquake. Tabas earthquake mapping (1978), PGA $913.61 \mathrm{~cm} / \mathrm{s} / \mathrm{s}$ is shown in Figure 5 a generated from earthquake records registered in the strongmotioncenter database. Figures $5 \mathrm{~b}$ to $5 \mathrm{c}$ depict the acceleration and displacement responses for the case where the slip load is obtained from the optimisation method and the case where the slip load is randomly selected.

Table 1 shows the results of the simulations for a 4-story structure for three cases, bending frame, friction damper without optimal slip load, and the friction damper with optimal slip load.

In the friction damper model, the optimisation method is solved by considering the ratio of acceleration value and allowable displacement (conditions $i$ and $i i$ ) equal to 0.3 and 0.25 . The value of $\xi_{a}$ and $\eta_{d}$ in the optimisation method will limit the slip load equal to $2000 \mathrm{kgf}$. As can be seen, the measured reduction ratio of the acceleration amplitude and displacement of the structure is 0.272 and 0.205 , respectively. Hence, the maximum amplitude of acceleration and displacement with friction damper at optimum slip load have been controlled compared to the non-friction damper structure.

As presented in Table 1, the acceleration and displacement amplitude ratio obtained from the optimisation method is 0.272 and 0.205 , respectively. Furthermore, this indicates that they are less than the conditions defined in the optimisation method and the method meets constraints. The frequency responses of acceleration and displacement of a four-story structure in an optimised and a non-optimised slip load are shown in Figure 6. As shown in Figure 6 , the number of peaks can be considerably controlled by using the friction damper in the structure and adjusting the slip load at the optimum level.

Moreover, to validate the results, further analyses were performed in 6 and 10-story structures with one and two bays. Figure 7 shows the acceleration and displacement responses of a 6-story structure that has been analysed by the proposed method. Further investigations have been carried out for 2-Bay structures under the earthquakes of Tabas, Manjil (1990- PGA: $128.21 \mathrm{~cm} / \mathrm{s} / \mathrm{s}$ ), and El-Centro (1940- PGA: $210.14 \mathrm{~cm} / \mathrm{s} / \mathrm{s}$ ), as can be seen in Tables 2-4.

One of the main concepts of the proposed method is to show its potential to apply to various structures. To this end, we addressed the details of the analysis method for 4 and

Table 1

Four story result under Tabas Earthquake, $\mathrm{S}=2000, \xi_{a}=0.30, \eta_{d}=0.25$

\begin{tabular}{lcc}
\hline Frame Type & Top Displacement $(\mathrm{cm})$ & Top Acceleration $\left(\mathrm{cm} / \mathrm{sec}^{2}\right)$ \\
\hline Bending & 18.0 & 1742 \\
Non-Optimum Frictional & 12.717 & 789.012 \\
Optimum Frictional & 3.69 & 475.171 \\
Obtained reduction ratio from optimisation method & 0.205 & 0.272 \\
\hline
\end{tabular}




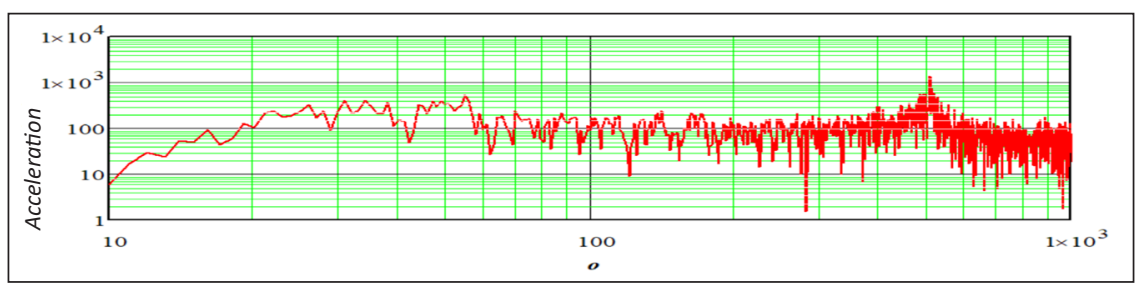

(a)

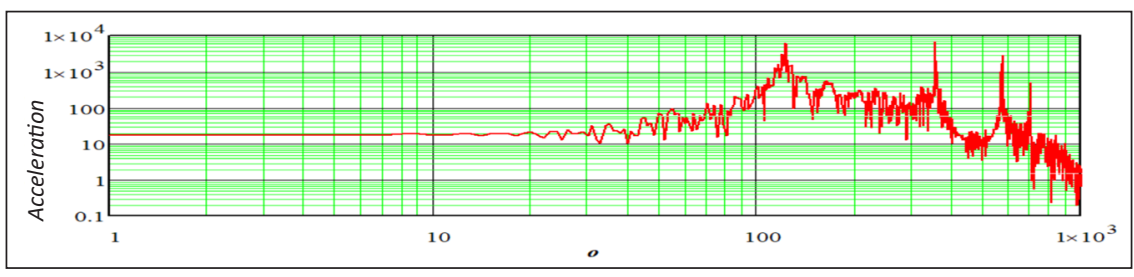

(b)

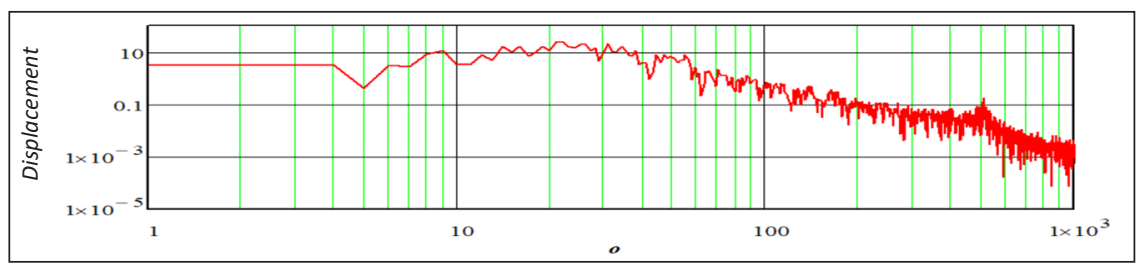

(c)

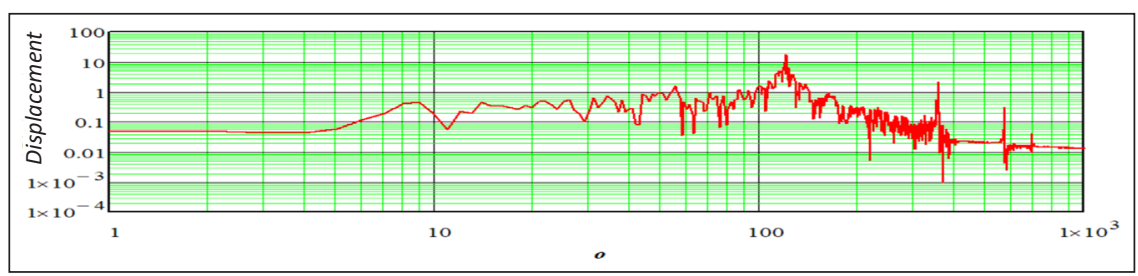

(d)

Figure 6. Frequency responses of a four-story building: (a) Acceleration with optimised slip load; (b) Acceleration without friction damper; (c) Displacement with optimised slip load; (d) displacement without friction damper

6 story structures in Figures 4 to 7. In each case, we compared the effect of non-optimum and optimum slip load on the response of acceleration and displacement. Then, simulations are extended to 2-Bay frames under different earthquakes. The results are shown in Table 2 to 4 . The results show no control of acceleration and displacement on the bending frame (without friction damper).

Similarly, for the non-optimum friction frame, we do not have adequate control. In the third frame, "optimum frictional", we can see the efficiency of the proposed method. That means the value of acceleration and displacement is reduced effectively compared to the bending frame. Since each earthquake has its specification and frequency spectrum, we have selected the most traditional ones in the world as a sample. As a result of the tectonic 
condition of Iran, Tabas and Manjil earthquakes are considered. Similarly, El-Centro is considered an external force to show no limit on applying the proposed method. As explained in Equation 8, we can apply various earthquakes as external forces. Therefore, the reduced motion equation is applicable for analysing the structure under various earthquakes.

In all simulations, the structural response per optimal value of slip load can can found. Hence, the simultaneous control of acceleration and displacement of the structure would

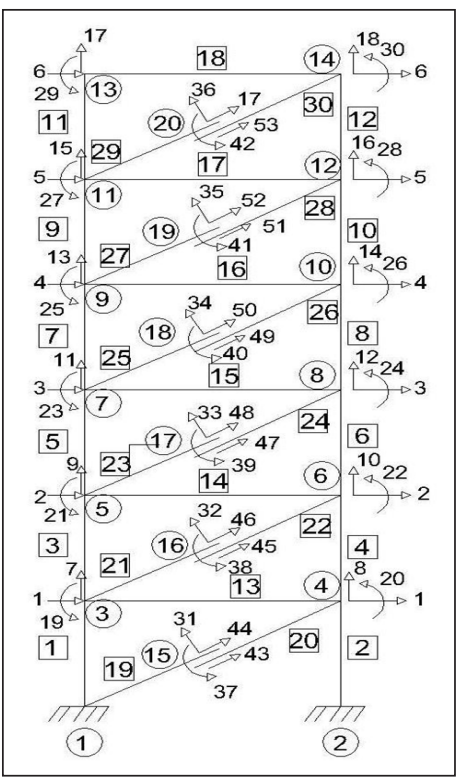

(a)

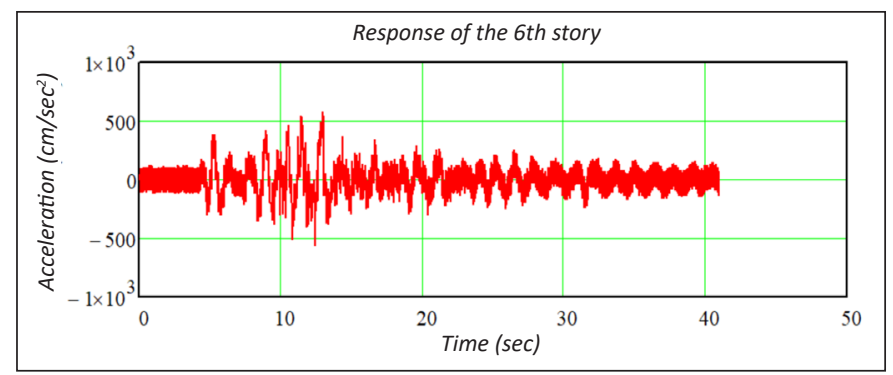

(b)

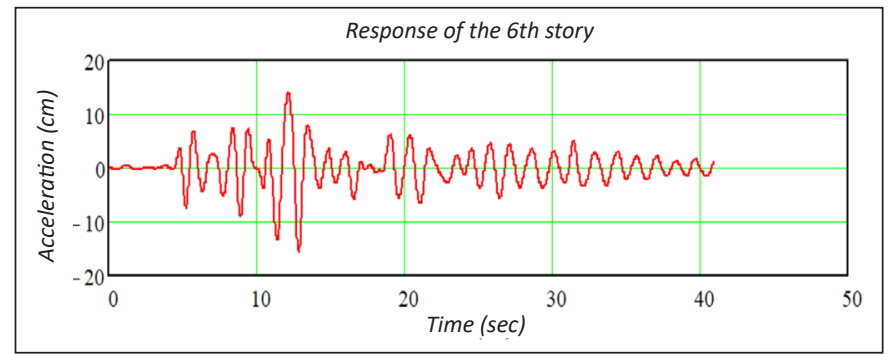

(c)

Figure 7. A six-story frame under Tabas earthquake s=1800kgf: (a) Analysed structure under proposed method; (b) Acceleration response; (c) Displacement response

Table 2

Six and Ten story results under Tabas $\xi_{a}=0.30$ and $\eta_{d}=0.25$

\begin{tabular}{llcc}
\hline Number of Stories & \multicolumn{1}{c}{ Frame Type } & Top Displacement $(\mathrm{cm})$ & Top Acceleration $\left(\mathrm{cm} / \mathrm{sec}^{2}\right)$ \\
\hline 6-story-1 Bay & Bending & 50.4 & 1792 \\
& Non-optimum Frictional & 15.6 & 954 \\
& Optimum frictional, S=1500 & 5.6 & 575 \\
6-story-2 Bay & Bending & 50.4 & 1956 \\
& Non-optimum Frictional & 16.7 & 954 \\
& Optimum frictional, S=2500 & 13.1 & 565 \\
10-story-1 Bay & Bending & 70.7 & 2126 \\
& Non-optimum Frictional & 44.4 & 954 \\
& Optimum frictional, S=2000 & 16.3 & 536 \\
& Bending & 86 & 3075 \\
& Non-optimum Frictional & 36.9 & 2391 \\
& Optimum frictional, S=2000 & 20.8 & 576 \\
\hline
\end{tabular}


Table 3

Four, Six, and Ten story results under Manjil $\xi_{a}=0.30$ and $\eta_{d}=0.25$

\begin{tabular}{llcc}
\hline \multirow{2}{*}{ Number of stories } & \multicolumn{1}{c}{ Frame Type } & $\begin{array}{c}\text { Top Displacement } \\
(\mathrm{cm})\end{array}$ & $\begin{array}{c}\text { Top Acceleration } \\
\left(\mathrm{cm} / \mathrm{sec}^{2}\right)\end{array}$ \\
\hline 4-story-1 Bay & Bending & 30.2 & 5325 \\
& Non-optimum Frictional & 12.7 & 1405 \\
& Optimum Frictional, S=1000 & 7.4 & 451 \\
4-story-2 Bay & Bending & 21.7 & 3560 \\
& Non-optimum Frictional & 10.8 & 843 \\
& Optimum Frictional, S=2000 & 4.7 & 437 \\
6-story-1 Bay & Bending & 30 & 2263 \\
& Non-optimum Frictional & 11.9 & 1316 \\
& Optimum Frictional, S=1500 & 7.2 & 439 \\
& Bending & 31.6 & 3895 \\
& Non-optimum Frictional & 18.5 & 1664 \\
10-story-1 Bay & 9 & 437 \\
& Optimum Frictional, S=2000 & 30.5 & 2160 \\
& Bending & 24.6 & 1162 \\
& Non-optimum Frictional & 12.6 & 384 \\
& Optimum Frictional, S=1500 & 28.9 & 3808 \\
& Bending & 18.4 & 890 \\
& Non-optimum Frictional & 14.4 & 506 \\
\hline
\end{tabular}

Table 4

Four, Six, and Ten story result under El-Centro $\xi_{a}=0.30$ and $\eta_{d}=0.4$

\begin{tabular}{llcc}
\hline Number of stories & Frame Type & $\begin{array}{c}\text { Top Displacement } \\
(\mathrm{cm})\end{array}$ & $\begin{array}{c}\text { Top Acceleration } \\
\left(\mathrm{cm} / \mathrm{sec}^{2}\right)\end{array}$ \\
\hline 4-story-1 Bay & Bending & 30.3 & 2714 \\
& Non-optimum Frictional & 7.7 & 1449 \\
& Optimum Frictional, S=1000 & 5.8 & 534 \\
4-story-2 Bay & Bending & 24 & 2683 \\
& Non-optimum Frictional & 8.4 & 1079 \\
& Optimum Frictional, S=2000 & 5.7 & 581 \\
6-story-1 Bay & Bending & 36.5 & 4462 \\
& Non-optimum Frictional & 20.2 & 1159 \\
& Optimum Frictional, S=1500 & 12 & 601 \\
6-story-2 Bay & Bending & 39.7 & 2734 \\
& Non-optimum Frictional & 19.2 & 1781 \\
& Optimum Frictional, S=2000 & 15.5 & 578 \\
10-story-1 Bay & Bending & 81.8 & 2633 \\
& Non-optimum Frictional & 34.7 & 1864 \\
& Optimum Frictional, S=1500 & 16.3 & 576 \\
10-story-2 Bay & Bending & 84.3 & 2353 \\
& Non-optimum Frictional & 46 & 1663 \\
& Optimum Frictional, S=2500 & 23.6 & 547 \\
\hline
\end{tabular}


be achieved. Figure 8 depicts the investigation results of changing the constraints in the optimisation method. By changing the values of $\xi_{a}, \eta_{d}$, the range of slip load can be determined to control the maximum amplitude and displacement values of the structure. The results illustrate that the optimum range of slip load of 1-bay structures is $1000 \mathrm{Kgf}$ to $2000 \mathrm{Kgf}$. On the contrary, for 2-bay structures, this range was increased, and the optimum range was $2000 \mathrm{Kgf}$ to $2500 \mathrm{Kgf}$. Thus, we can find the optimum slip load in each area with various allowable thresholds of acceleration and displacement.

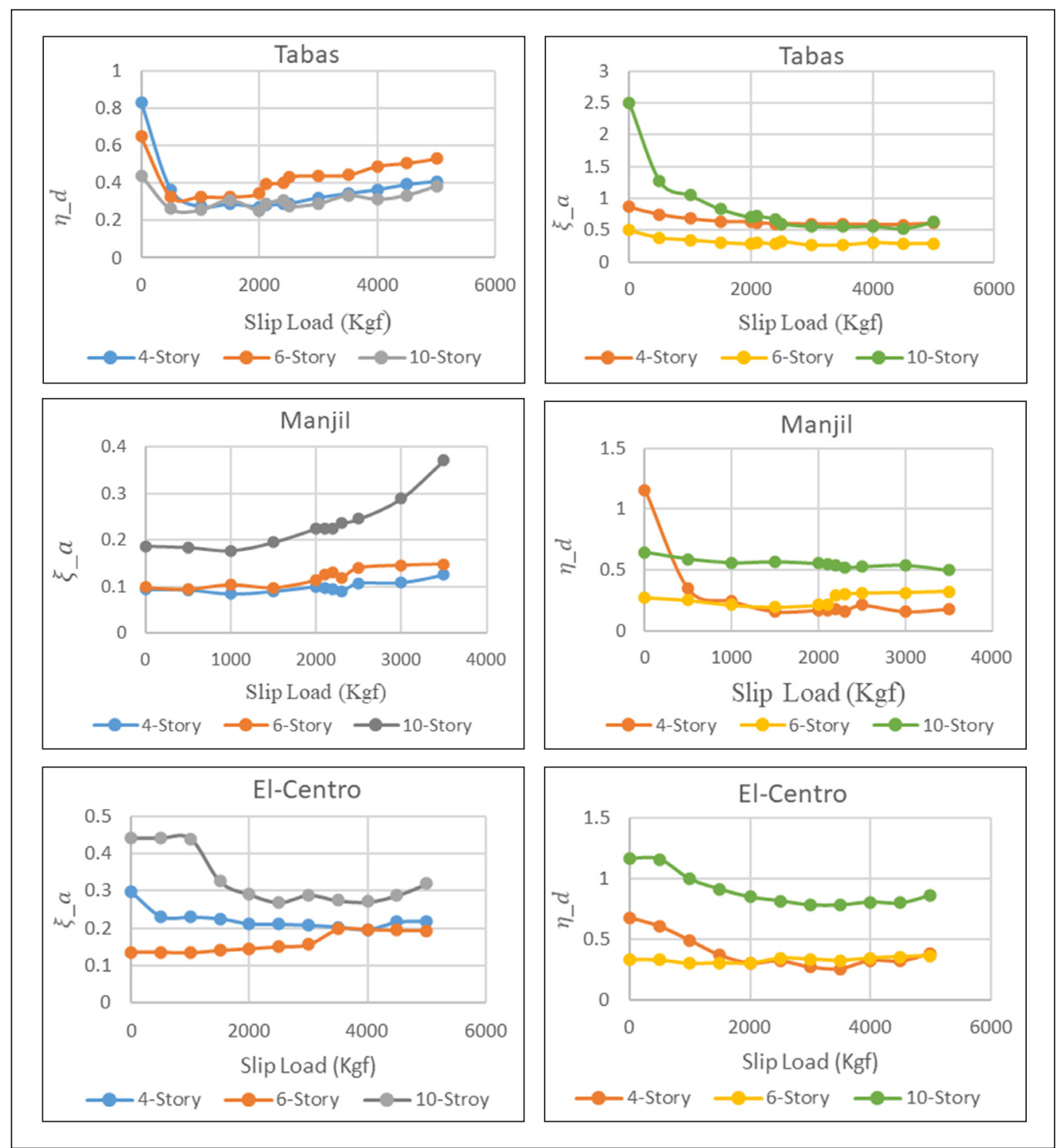

Figure 8. Variation of optimisation method constraints vs. slip load for 4, 6, and 10 story buildings under Tabas, Manjil, and El-Centro earthquakes 
Table 5

Comparison of the proposed method with other references

\begin{tabular}{|c|c|c|c|c|}
\hline Ref & $\begin{array}{c}\text { Structure under } \\
\text { Test }\end{array}$ & $\begin{array}{l}\text { Displacement } \\
\text { Reduction (\%) }\end{array}$ & $\begin{array}{c}\text { Acceleration } \\
\text { Reduction }(\%)\end{array}$ & $\begin{array}{l}\text { Inter Story Drif } \\
\text { Reduction }(\%)\end{array}$ \\
\hline Lee et al, 2008 & 6-story & - & 31.8 & 66 \\
\hline This paper & & 80.1 & 80.3 & 84.2 \\
\hline Fallah \& Honarparst, 2013 & 4-Story & 40.3 & 19.6 & 35 \\
\hline This paper & & 80.1 & 80.3 & 84.2 \\
\hline Feliciano, 2015 & 6-story & 69 & 71 & - \\
\hline This paper & & 68 & 86 & 70.1 \\
\hline Miguel \& Lopez, 2018 & 4-story & 82.73 & - & 81.8 \\
\hline This paper & & 80.1 & 80.3 & 84.2 \\
\hline Domenico, 2020 & 4-story & 60 & 40 & - \\
\hline This paper & & 80.1 & 80.3 & 84.2 \\
\hline Sanghai \& Pawade, 2021 & 6-story & - & - & 33 \\
\hline This paper & & 68 & 86 & 70.1 \\
\hline Miguel \& Pérez, 2017 & 9-story & 72 & 70 & 83.3 \\
\hline This paper & & 81 & 80 & 86.2 \\
\hline Lopez \& Miguel, 2015 & 10 -story & 66 & - & 76 \\
\hline This paper & & 80.07 & 78.12 & 79 \\
\hline Kim \& An, 2016 & 4-story & 30 & - & 40 \\
\hline This paper & & 80.1 & 80.3 & 84.2 \\
\hline Palacios et al. 2020 & 4-story & - & 40 & 50 \\
\hline This paper & & 80.1 & 80.3 & 84.2 \\
\hline
\end{tabular}

Table 5 shows a comparison between the results of this paper and other studies. The reduction ratio depicts how much the acceleration and displacement amplitudes can be controlled using optimised slip load compared to non-friction damped structures.

Furthermore, to assess the proposed MAHA and other investigations, 4, 6, 9, and 10 -story buildings are analysed with the same situations as mentioned previously. Table 5 compared the result of a 4-story with the findings of Miguel and Lopez (2018), which shows that the proposed method of this paper can improve all three parameters above $80 \%$ simultaneously. Lopez and Miguel (2015) showed a 43\% reduction of inter-story drift for a 6-story buildings, while this paper achieved $70 \%$.

Lee H. et al. (2008) investigated un-damped and damped frames. They achieved a $31.8 \%$ and $43 \%$ reduction of maximum acceleration for 6-story and 4-story buildings, respectively. Also, they reported a 66\% reduction of inter -story drift. Prez et al. (2017a) reported a 54\% reduction in acceleration in controlled mode compared to uncontrolled. Miguel and Lopez (2018) resulted in an 80\% reduction of acceleration and inter-story drift for a 4-story building. Sanghai \& Pawade (2021) and Miguel \& Pérez (2017(, approved 


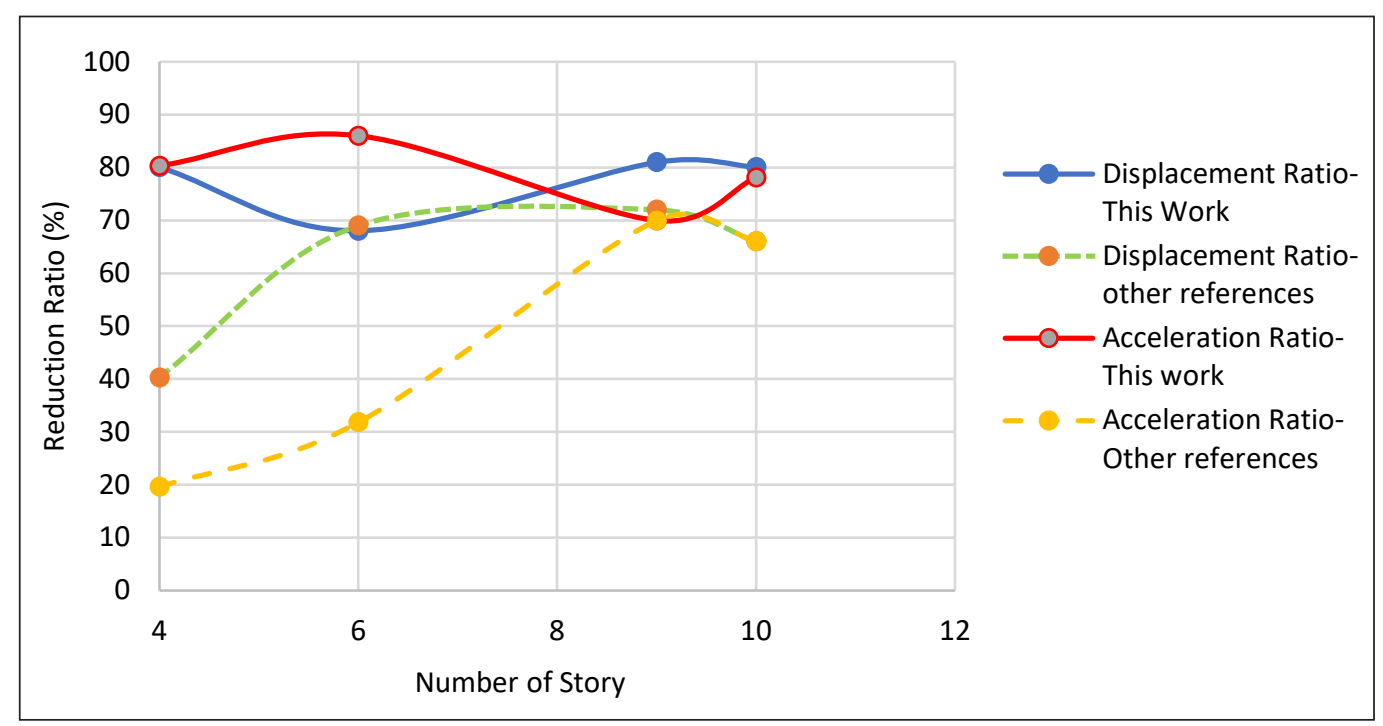

Figure 9. Comparison of results of this study with other references (Table 5)

that the displacement reductions are about $80 \%$ for 4,9 , and 10 stories scenarios. Palacios et al. (2020) reported about 50\% and 40\% reductions in the overall inter story-drift and acceleration peak-value, respectively. Kim \& An (2016) concluded that maximum roof displacement and the inter-story drift ratio were reduced by $30 \%$ and $40 \%$, respectively.

Moreover, to assess the effectiveness of the proposed method, numerical results are presented for 6, 9, and 10 stories. As can be seen in Table 5, the analogy of Sanghai \& Pawade (2021), Miguel and Pérez (2017), and Lopez and Miguel (2015) approved that the displacement reductions of $82 \%$ and $80 \%$ can be achieved for 4,9 , and 10 -stories scenarios, respectively. Regarding the acceleration, the reduction ratios are $80 \%$ and $78 \%$. Also, the inter-story drift is investigated. It is under control in such a way that it is about $70 \%$ for all investigated structures.

The results of present work with the mentioned references has been comprised Table 5 and illustrated in Figure 9 to better depict the performance of the proposed optimisation method. . Acceleration reduction ratios, based on this study, are higher than other references for various buildings. Moreover, the results for displacement reduction ratio for various investigated buildings show that the proposed method is more efficient than other references.

There are no height limitations and 4, 6, 9, and 10 stories are considered a prototype. As seen in Equations 2, 3, 17, 18, 19, and 20, the analysis is parametric, and the method is applicable for various numbers of stories $\left(n_{\text {story }}\right)$. In fact, by increasing the number of buildings, the degree of freedom (DOFs) and relevant matrix dimension would be regenerated based on that building. However, it is worth noting that for tall buildings, there are specific considerations. 


\section{CONCLUSION}

This paper proposed an intelligent control system that enabled simultaneous control of acceleration and displacement in a friction damped structure. The functionality of the proposed system was formulated as an optimisation method. A combined analysis approach named MAHA was presented to solve the problem, combining HL-MAFD and SGA's heuristic algorithm. Based on the results of this study, the following conclusions may be drawn:

- By using the proposed intelligent control system, the optimal value of slip load could be found that resulted in considerable control of acceleration and displacement of structures. The obtained results at these optimum slip loads showed the displacement reduction of $82 \%$ and $80 \%$ for $4,6,9$, and 10 story structures, respectively. Regarding the acceleration, the reduction ratio is $80 \%$ and $78 \%$. Also, the inter-story drift was investigated. It was under control in such a way that it was about $71 \%$ to $86 \%$ in all mentioned structures.

- The proposed approach was designed parametric and could be applied to various structures under different types of earthquakes. The numerical simulations concluded that the control system could be easily adjustable by changing optimisation method's acceleration and displacement ratio threshold.

- The proposed approach would be extended to tall and high-rise buildings regarding the high rise design considerations.

- By analysing the responses of structures under optimum slip load, the intelligent control system gives a statistical view of the behaviour of structures during the designing phase. The engineers could find the controlled responses of structures on that area and design a suitable friction damper based on the optimum slip load. Thus, this would be more beneficial in strengthening structures against earthquake damages in each specific area.

- The proposed control system would reduce the financial loss because of oversizing by allowing the slip load of friction damper to be designed based on the particular structure and area.

The proposed methodology is recommended as an effective and economical tool for controlling the damage of structures.

\section{ACKNOWLEDGEMENT}

The authors acknowledge the support of the Islamic Azad University of Central Tehran Branch. 


\section{REFERENCES}

Apostolakis, G., \& Dargush, G. (2010). Optimal seismic design of moment-resisting steel frames with hysteretic passive devices. Earthquake Engineering and Structural Dynamics, 39(4), 355-376. https:// doi.org/10.1002/eqe.944

Bhaskararao, R., \& Jangid, S. (2006). Seismic analysis of structures connected with friction dampers. Journal of Engineering Structures, 28(5), 690-703. https://doi.org/10.1016/j.engstruct.2005.09.020

Domenico, D., Ricciardi, G., \& Zhang, R. (2020). Recent aadvances in the design of structures with passive energy dissipation systems. Journal of Applied Sciences, 10, 1-6. https://doi.org/10.3390/app10082819

Fallah, N., \& Honarparst, S. (2013). NSGA-II based multi objective optimization in design of pall friction dampers. Journal of Constructional Steel Research, 89, 75-85. https://doi.org/10.1016/j.jcsr.2013.06.008

Fateh, A., Hejazi, F., Ramanathan, R., \& Jaffar, S. (2016). Seismic response of a light rail transit station equipped with braced viscous damper. Pertanika Journal of Science \& Technology, 24(2), 273-283.

Feliciano, C. (2015). Design optimization for plane structures equipped with friction dampers. Institute Superior Tecnico, Lisboa, Portugal, 1-10.

Kim, J., \& An, S. (2016). Optimal distribution of friction dampers for seismic retrofit of a reinforced concrete moment frame. Advances in Structural Engineering, 20(10), 1523-1539. https://doi. org/10.1177/1369433216683197

Lee, H., Park, H., Lee, K., \& Min, K. W. (2008). Allocation and slip load of friction dampers for a seismically excited building structure based on story shear force distribution. Engineering Structures, 30(4), 930-940. https://doi.org/10.1016/j.engstruct.2007.03.020

Lee, S., Park, J., Moon, B., \& Min, K. (2008). Design of bracing-friction damper system for seismic retrofitting. Smart Structures and Systems, 4(5), 685-696. https://doi.org/10.12989/sss.2008.4.5.685

Logan, D. (2007). A first course in the finite element method. Nelson Publishing.

Lopez, S., \& Miguel, L. (2015). A firefly algorithm for the design of force and placement of friction dampers for control of man-induced vibrations in footbridges. Journal of Optimization and Engineering, 16(3), 633-661. https://doi.org/10.1007/s11081-014-9269-3

Majd, A., Damerji, H., Hallal, J., \& Fakih, M. (2019). Effectiveness of friction dampers on the seismic behavior of high rise building vs shear wall system. Engineering Reports, 1(5), 1-14. https://doi.org/10.1002/ eng2.12075

Miguel, F., \& Lopez, R. (2018). Methodology for the simultaneous optimization of location and parameters of friction dampers in the frequency domain. Engineering optimization, Tailor and Francis Journal, 50(12), 2108-2122. https://doi.org/10.1080/0305215X.2018.1428318

Miguel, F., \& Pérez, S. (2017). Optimization of location and forces of friction dampers. REM International Engineering Journal, 70(3), 273-279. https://doi.org/10.1590/0370-44672015700065

Nizic, A., \& Mestrovic, D. (2011). Seismic dampers in engineering structures. Journal of the Croatian Association of Civil Engineering, 63(7), 661-667. 
Palacios, F., Masswgu, J., Rossel, M., \& Karimi, H. (2020). Distributed passive actuation schemes for seismic protection of multibuilding systems. Journal of Applied Science, 10(7), 1-30. https://doi.org/10.3390/ app10072383

Pall, S. (1996, June 23-26). Friction-dampers for seismic control of buildings a Canadian experience. In Proceeding of the World Conference on Earthquake Engineering (pp. 1-8). Montreal, Canada.

Pasquin, C. (2004, August 1-6). Friction dampers for seismic rehabilitation of eaton's building. In Proceeding of the World Conference on Earthquake Engineering (pp. 1-10). Vancouver, Canada.

Perez, S., \& Miguel, L. (2017a). A new assessment in the simultaneous optimization of friction dampers in plane and spatial civil structures. Journal of Mathematical Problems in Engineering, 2017, Article 6040986. https://doi.org/10.1155/2017/6040986

Perez, S., \& Miguela, F. (2017b). Robust simultaneous optimization of friction damper for the passive vibration control in a Colombian building. Journal of Procedia Engineering, 199, 1743-1748. https:// doi.org/10.1016/j.proeng.2017.09.430

Rashidi, H., Khanlari, K., Zarfam, P., \& Ashtiany, M. G. (2020). A novel approach of active control of structures based on the critically damped condition. Journal of Vibration and Control, O(0), 1-13. https:// doi.org/10.1177/1077546320944300

Sanghai, S., \& Pawade, P. (2020). Effectiveness of friction dampers on seismic response of structure considering soil-structure interaction. Journal of the Croatian Association of Civil Engineering, 72(1), 33-44. https:// doi.org/10.14256/JCE.1982.2017

Sanghai, S., \& Pawade, P. (2021). Optimal placement of friction dampers in building using considering nonlinearity of soil. Springer Journal of Innovative Infrastructure Solutions, 6(28), 1-18. https://doi. org/10.1007/s41062-020-00395-8

Shaw, S. (1986). On the dynamic response of a system with dry friction. Journal of Sound and Vibration, 108(2), 305-325. https://doi.org/10.1016/S0022-460X(86)80058-X 


\section{APPENDIX}

\section{Supplementary Data}

To analyse the behaviour of the structure using the proposed method, we need to form a reduced general equation of motion as mentioned in Equation 8. To this end, we need to calculate the system stiffness matrix accurately. In the following, the analysis steps for a four-story structure with a frictional damper are addressed. The frame structure has been shown in Figure 4. The nodes matrix is shown in Equations 11 and 12. The number of elements $(N E L)$ is 20 , and the total number of degrees of freedom $(N D O F)$ is 36 .

$$
\begin{aligned}
& \text { NEL }=20, \text { DOF }=e+i=36, \mathrm{e}=4, i=32 \\
& \text { Nodes }=\left(\begin{array}{ccccccccccccccc}
0 & 400 & 0 & 400 & 0 & 400 & 0 & 400 & 0 & 400 & 200 & 200 & 200 & 200 \\
0 & 0 & 300 & 300 & 600 & 600 & 900 & 900 & 1200 & 1200 & 150 & 450 & 750 & 1050
\end{array}\right)
\end{aligned}
$$

Also, elements matrix is (Equation 13):

$$
\text { Elements }=\left(\begin{array}{cccccccccccccccccccc}
1 & 2 & 3 & 4 & 5 & 6 & 7 & 8 & 3 & 5 & 7 & 9 & 1 & 11 & 3 & 12 & 5 & 13 & 7 & 14 \\
3 & 4 & 5 & 6 & 7 & 8 & 9 & 10 & 4 & 6 & 8 & 10 & 11 & 4 & 12 & 6 & 13 & 8 & 14 & 10
\end{array}\right)
$$

The degree of freedom matrix and the member code matrix are the following (Equation 14 and 15):

$$
\begin{aligned}
\mathrm{DOF} & =\left(\begin{array}{cccccccccccccc}
0 & 0 & 1 & 1 & 2 & 2 & 3 & 3 & 4 & 4 & 29 & 31 & 33 & 35 \\
0 & 0 & 5 & 6 & 7 & 8 & 9 & 10 & 11 & 12 & 21 & 22 & 23 & 24 \\
0 & 0 & 13 & 14 & 15 & 16 & 17 & 18 & 19 & 20 & 25 & 26 & 27 & 28
\end{array}\right) \\
M C M^{i} & =\operatorname{stack}\left(\text { DOF }^{\text {member }_{2, i},}, \text { DOF }^{\text {member }_{3, i}}\right)
\end{aligned}
$$

The total stiffness matrix of the structure that needs to solve the general equation of motion would be obtained via Equation 16:

$$
\boldsymbol{K}_{\text {sys }}=\sum_{i=1}^{N E L} T_{i}^{T} R_{i}^{T} k_{i} R_{i} T_{i}
$$

Also, $k_{i}$ is the member's stiffness matrix in rank of $6 \times 6$ (Logan, 2007). $K_{m m}, K_{m s}, K_{s s}, K_{s m}, K_{s s}$ are submatrices of $K_{s y s}$ and defined as Equation 17-20:

$$
\begin{aligned}
& K_{m m}=\operatorname{submatrix}\left(K_{\text {sys }}, 1, n_{\text {story }}, 1, n_{\text {story }}\right) \\
& K_{m s}=\operatorname{submatrix}\left(K_{\text {sys }}, 1, n_{\text {story }}, n_{\text {story }}+1, D O F\right) \\
& K_{s m}=\operatorname{submatrix}\left(K_{\text {sys }}, n_{\text {story }}+1, D O F, 1, n_{\text {story }}\right) \\
& K_{s s}=\operatorname{submatrix}\left(K_{\text {sys }}, n_{\text {story }}+1, D O F, n_{\text {story }}+1, D O F\right)
\end{aligned}
$$

Finally, the $\boldsymbol{K}$ matrix is obtained according to Equation 6 b. 
